\title{
Medical Interpreting at the Tokyo 2020 Olympic and Paralympic Games
}

\author{
NAOKO ONO*1) 2) \\ *1) Graduate School of Medicine, Juntendo University, Tokyo, Japan, \\ *2) Faculty of International Liberal Arts, Juntendo University, Tokyo, Japan
}

\begin{abstract}
According to an estimate of the Japan National Tourism Organization, 2,930,000 overseas visitors came to Japan in April 2019. With the growing the number of non-Japanese and Tokyo hosting the 2020 Summer Olympics and Paralympics, there is a rising need in Japan for international medical communication. Medical interpreting training is urgently required owing to language problems through the rapid increase in the number of non-Japanese workers in Japan over recent decades. A systematic review indicated five core competencies for medical interpreters: maintaining accuracy and completeness; medical terminology and understanding the human body; behaving ethically and making ethical decisions; nonverbal communication skills; and cross-cultural communication skills. A training course to develop those skills was implemented in Juntendo University's Faculty of International Liberal Arts (FILA). Since its founding in 1838, Juntendo University has produced experts in medicine, health care, and sports. The rich culture accumulated over that time has been adapted for the global era: the university offers a progressive education that simultaneously teaches internationalism and currently required health expertise. The FILA offers several courses to help promote medical interpreters. A practical example is presented in this paper to show the capabilities of the FILA system. Interfaculty cooperation is needed to bridge the gap between medical professionals and patients with limited Japanese proficiency. By nurturing medical interpreters, Juntendo University is expected to promote interfaculty collaboration in supporting non-Japanese patients. In 2020, Japan should create an environment in which people from overseas can visit the country without worrying about medical care.
\end{abstract}

Key words: medical interpreting, medical interpreters, Olympics, Paralympics, Tokyo 2020

\section{Introduction}

\section{Increasing number of non-Japanese in Japan}

According to an estimate of the Japan National Tourism Organization ${ }^{1)}, 2,930,000$ overseas visitors came to Japan in April 2019. A survey of the Statistics Bureau, Ministry of Internal Affairs and Communications revealed that the proportion of nonJapanese in the country exceeded $1 \%$ in $2000^{2)}$. In 2018, Japan's Diet passed an immigration bill that would allow about 340,000 non-Japanese to work in Japan over the subsequent 5 years. With the increase in the number of non-Japanese and Tokyo hosting the 2020 Summer Olympics and Paralympics, there is growing need for international language communication in medical settings in Japan. Further, medical tourism has emerged as a new area of business. Japan's government aims to increase the number of overseas visitors to the country to 20 million by 2020. Medical tourism is regarded as one growth area for Japan. Against this background, language barriers put the health of non-Japanese at risk owing to difficulty in accessing health care and communicating with medical professionals. Especially during the Tokyo 2020 Olympic and Paralympic Games, many foreigners

\footnotetext{
Naoko Ono

Graduate School of Medicine, Juntendo University

2-1-1 Hongo, Bunkyo-ku, Tokyo 113-8421, Japan

TEL: +81-3-3813-3111 (ext: 2993) E-mail: na-ono@juntendo.ac.jp

〔Received Nov. 30, 2019〕〔Accepted Jan. 23, 2020〕
}

Copyright (C) 2020 The Juntendo Medical Society. This is an open access article distributed under the terms of Creative Commons Attribution License (CC BY), which permits unrestricted use, distribution, and reproduction in any medium, provided the original source is properly credited. doi: 10.14789/jmj. 2020.66.JMJ19-P06 
unused to Japanese culture will visit the country: if they become ill or injured, many problems could arise. Research has underlined the difficulties that language barriers can present in a medical context ${ }^{3)}$. There is an urgent need for medical interpreting training in Japan owing to language problems that have resulted from the rapid increase in the number of foreign workers over recent decades. Owing to the limited practical activities related to medical interpreting, it is difficult to acquire appropriate interpreting skills. Medical interpreters are needed to bridge the communication gap between medical professionals and patients with limited Japanese proficiency.

\section{Interpreting needs at international sports events}

A fact sheet on previous Olympic Games ${ }^{4)}$ detailed how many athletes and staff took part in the events. Over 10,000 athletes from 199 nations participated in the 2000 Summer Olympics in Sydney ${ }^{5)}$. According to the same fact sheet, 10,568 athletes competed and more than 70,000 volunteers provided support at the London Summer Olympics in 2012 ${ }^{4)}$. Many factors are important for a successful Olympics. However, one key issue is effective communication: interpreters play a very important role in assisting communication among people from different countries.

\section{Interpreting at Olympics and Paralympics}

Ono ${ }^{6)}$ conducted a literature survey and interviews to determine how interpreters worked at previous Olympics and Paralympics. The literature survey covered articles in newspapers, published journals, and on Web sites from 2008 to 2014; the articles were identified using an Internet search for "Olympic interpreters." The literature survey revealed that there were both professional interpreters (divided into the interpreter to translate the speaker's words while the speaker is talking, and 'listen, pause, talk' interpreters) and volunteer language assistants at the Sydney Games, as examined by $\mathrm{Yue}^{7)}$. According to Nishikawa ${ }^{8)}$, at the London Games, official interpreters worked only in certain settings; by contrast, volunteer interpreters (called "Game Makers" at the London
Games: they helped make the events run smoothly) played many roles other than interpreting. Nishikawa ${ }^{8)}$ observed, "Game Makers were assigned more than 860 jobs, including setting up values, helping athletes in the athletes' village, performing dance at ceremonies."

Ono ${ }^{6)}$ interviewed a professional interpreter who worked in English and Japanese; he was employed for the London Olympics as an interpreter working for a nationwide broadcasting company. His main job was liaising with film crews for filming events, interviewing participants, and corresponding with sponsors. The interpreter stated that when interpreting for the Olympics, he did not believe that knowledge or terminology was more difficult or specialized than for other occasions. However, when asked to describe impressive aspects of interpreting in the Olympics, he replied that it was "very special to meet and interpret for elite athletes and coaches" and he was able to "also get access to privileged areas and get to see how the games function from close up."

\section{Medical interpreting at the Olympics and Paralympics}

In addition to services for the sports events, interpreting for the Tokyo Games will require medical interpreters: the events will take place in summer, and participants and spectators may suffer from heatstroke and other conditions. According to the Web site of the Ministry of Health, Labour and Welfare (MHLW $)^{9)}$, medical interpreting and multilingual medical interview sheets have been prepared. However, to accommodate future increases in visitors and workers from overseas, it is necessary for Japan to train medical interpreters.

A research team under the MHLM set up a licensing system preparation committee in the International Society of Clinical Medicine, which aims to launch a licensing system for medical interpreters in 2020. Toward raising the quality of medical interpreting, the program will provide certification to medical interpreters who can work in Japanese and other languages if they pass written and oral tests. Applicants will be tested on their knowledge of medical terminology, cultural

This manuscript was submitted for the Special Issue "Tokyo 2020 Olympic and Paralympic Games, and Sportology", prior to the decision to postpone the Tokyo 2020 Summer Olympics to 2021. 


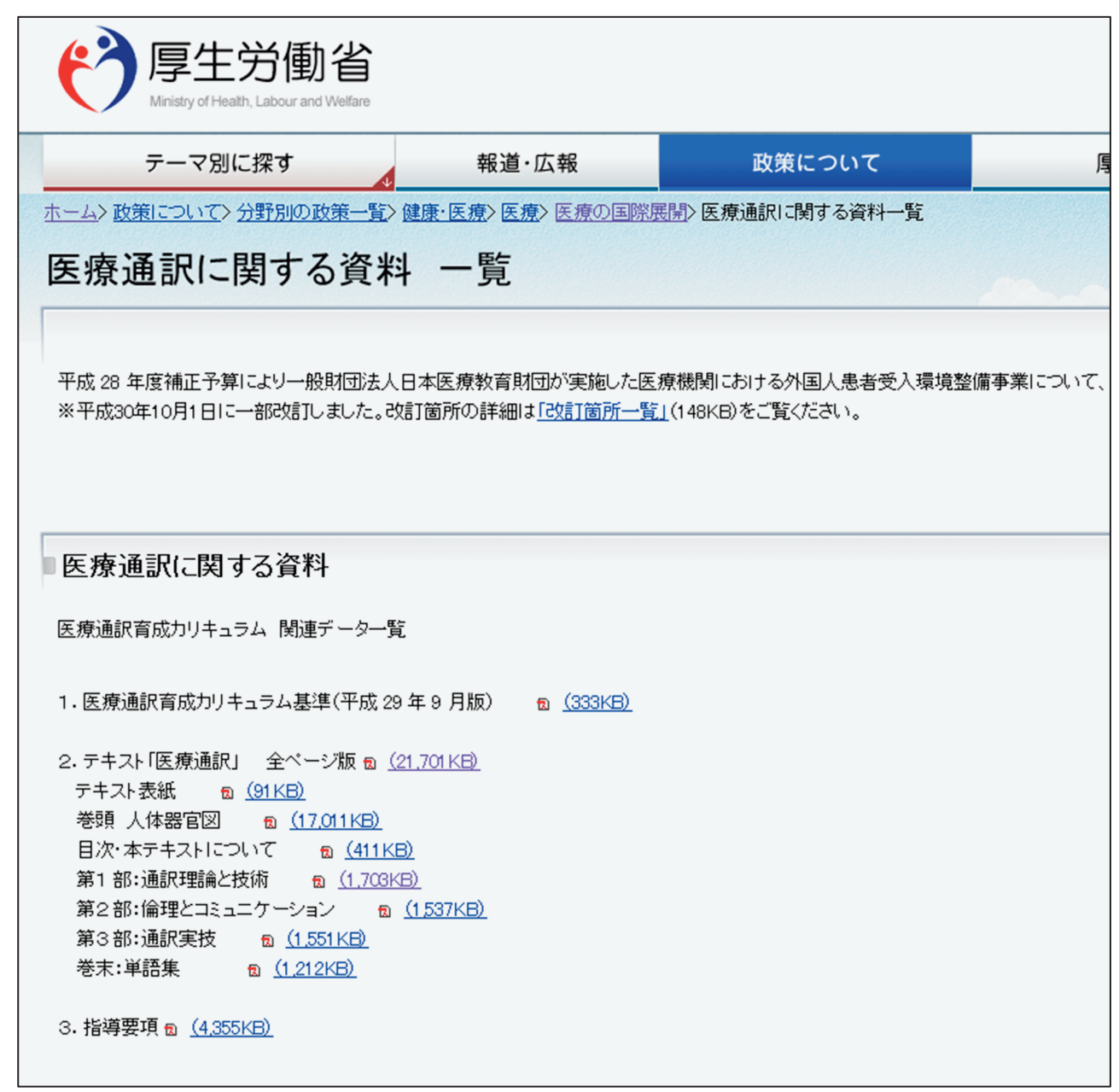

Figure-1 References for medical interpreting on the Web site of the Ministry of Health, Labour and Welfare

differences, and work ethics. Currently, many interpreters are either certified under programs offered by medical interpreting organizations or working as volunteers without certification. Not many medical interpreters at present have undergone dedicated training.

Preparations are under way to accommodate a future increase in non-Japanese visitors and workers by training English-speaking medical staff and paramedics at hospitals in Tokyo. The MHLW has unveiled guidelines and materials for training future medical interpreters (Figure-1). The guidelines were prepared under the auspices of the Project for Improving the Environment for Accepting Foreign Patients at Medical Institutions of the MHLW. The main content is as follows: theory and practice in medical interpreting; ethics and communication; knowledge required for medical interpreters; and practical training for medical interpreters. The guidelines are currently applied in medical interpreting. Some graduate schools (such as Osaka University) have courses that comply with the guidelines. After having completed the course (of over 100 hours) in accordance with the guidelines, prospective medical interpreters are able to take an examination to qualify as a certified medical interpreter. Upon completing an additional 3-hour training session, the individual can become recognized as a certified interpreter by the International Society of Clinical Medicine.

Ono ${ }^{10)}$ conducted a literature search and identified five core competencies for medical interpreters: (1) maintaining accuracy and completeness; (2) medical terminology and understanding the human body; (3) behaving ethically and making ethical decisions; (4) nonverbal communication skills; and (5) cross-cultural communication skills. The following is an overview of the results with those categories. 


\section{Maintaining Accuracy and Completeness}

The studies cited below reported that errors in medical interpretation were likely to trigger negative effects such as interference with the transfer of information, reduced emotional support, and diminished rapport. Untrained interpreters tended to make more mistakes than professional medical interpreters. This suggests that maintaining accuracy and completeness is a core competency for professional medical interpreters. Gany et $a l .{ }^{11)}$ compared the errors made by trained and untrained interpreters. The trained interpreters had each received 80 hours of medical interpreting training at the Center for Immigrant Health, New York University School of Medicine; their training was similar to that offered at other US institutions. To identify errors related to clinical practice (hereinafter "clinical errors"), their medical encounters were recorded and transcribed. It was found that trained interpreters were less likely to make clinical errors than untrained ones. Gany et al. also determined that the use of longer sentences led to greater errors in interpretation. Pham et al. ${ }^{12}$ audiotaped 10 conferences attended by medical professionals. The authors identified alterations, additions, omissions, substitutions, and editorializations with medical interpreting: 93\% of those occurrences were likely to produce a negative effect.

\section{Medical Terminology and Understanding the Human Body}

Health-related knowledge and medical vocabulary are a core competency in medical interpreting. Sang-Bin ${ }^{13)}$ discussed medical interpreting in South Korea with the focus on socio-legal and training aspects. The author identified core curricular areas: role and ethics; basic interpreting techniques; controlling the flow of the session; health-care practice and medical terminology; professional development; and impact of culture.

\section{Behaving Ethically and Making Ethical Decisions}

To maintain their professional credibility, medical interpreters have to recognize their role in a medical setting and behaving in accordance with that role. White and Laws ${ }^{14)}$ audiotaped and analyzed 13 consultations. They found that uncertified hospital interpreters engaged in role exchange by assuming the provider's role or the patient's role; in the case of pediatric patients, such interpreters even socialized with mothers. Interpreters have to maintain professional boundaries: they cannot become personally involved with medical professionals, patients, or patient family members who may work with them. For the interpreter, this means remaining responsible for one's actions, staying organized, and being honest in all communications in interpretation settings. To behave ethically and make ethical decisions, interpreters have to keep their focus on health care-regardless of any personal biases that may arise-and must not present their own opinions. Interpreters have to convey messages between patients and medical professionals even if they disagree with some matters.

\section{Nonverbal Communication Skills}

Some qualitative studies have examined the factors for interpreters when communicating with medical professionals and patients. The results showed that nonverbal communication is important for effective communication. Espondaburu ${ }^{15)}$ reviewed the literature on communication techniques practiced by doctors when delivering bad news. The author emphasized the importance of the medical interpreter's tone of voice and pace; she argued that nonverbal communication should not be overlooked. Espondaburu also determined that the medical interpreter's nonverbal cues were an important means of creating a compassionate environment. Pugh and Vetere ${ }^{16)}$ conducted a qualitative study to explore mental health professionals' experience with empathy in clinical work when dealing with an interpreter. The authors conducted semi-structured interviews among mental health professionals who regularly worked with interpreters. The study suggested that medical interpreters should be sensitive to a patient's nonverbal communication.

\section{Cross-cultural Communication Skills}

In cross-cultural communication, several studies 
have demonstrated that skills in cross-cultural communication are indispensable. Cross and Bloomer ${ }^{17)}$ examined the communication mechanisms adopted by mental health clinicians to determine how they modify their communications to reconcile cultural differences and promote self-disclosure. The authors investigated 53 clinicians dealing with seven groups of culturally and linguistically diverse patients and asked them about their perceptions in communicating. Two particular areas of concern emerged: respect and cultural understanding. The study mainly addressed clinicians, but the results are partially applicable to medical interpreters.

Larrison et al. ${ }^{18)}$ performed an interview survey of 17 hospital staff members and 30 Latino patients. The authors found that when the interpreter showed commitment to the Latino community, effective communication resulted.

Norris $e t a l .{ }^{19)}$ conducted a qualitative study with four focus groups consisting of 43 professional medical interpreters. The authors applied grounded theory and developed three frameworks for understanding high-quality language-discordant communication about end-of-life care: professionalism, including emotional support capability; communication skills and cultural sensitivity; and the role conflict between strict interpretation and acting as a cultural broker. Karliner et al. ${ }^{20)}$ observed that when medical practitioners lacked knowledge of a patient's culture, it hindered their ability to provide quality medical care.

\section{Juntendo University's Faculty of International Liberal Arts}

\section{Overview}

In April 2015, Juntendo University's Faculty of International Liberal Arts (FILA) was established in Ochanomizu, central Tokyo. Since its founding in 1838, Juntendo University has produced experts in medicine, health care, and sports. The rich cultural buildup over that time has been adapted for the global era: the university offers a progressive education that simultaneously teaches internationalism and currently required health expertise. The FILA strives to cultivate truly global citizens who resolve problems with a global perspective: it utilizes the spirit of jin (benevolence) and covers topics on social issues, communication across cultures, and health.

Rather than simply attempting to face the problems occurring in the world, FILA students strive to understand the reasons behind them, what is necessary to solve them, and who it is they are really trying to help. FILA students develop the ability to think of others based on the spirit of jin. The FILA mission is to lend its full support to those who seek to help others in need, no matter where they are. The educational goal of the FILA is cultivating global citizenship that can contribute to sustainable development. For the future of humanity to be sustainable, the FILA regards the development of diversity as critical; global citizens who can contribute to the development of a multicultural and multilingual society are required. The FILA seeks students who possess the qualities necessary to become global citizens who contribute to a multicultural, symbiotic society.

\section{FILA preparations for medical interpreting at} Tokyo 2020

1) Basic foreign language course

All FILA students have to sit the TOEFL test twice a year, and freshmen learn a language other than English (Chinese, French, or Spanish) as a compulsory subject. Foreign language courses are taught with small class sizes. For example, the required English courses taken during the first 2 years are limited to 20 students per class. In addition to care and support for each student, the classes are highly regarded for their collaborative learning approach, whereby students can help one another while learning proactively. Through discussions, presentations, and other activities in foreign languages, the students learn intercultural communication skills that go beyond just language learning.

\section{2) Overseas experience}

The FILA offers training and study opportunities that provide a deep intercultural experience in other countries. The extensive overseas training program gives students the chance to apply what they learned in their daily studies. The FILA offers a rich list of programs tailored to meet students' interests and concerns.

One such program is the Global Health Training 


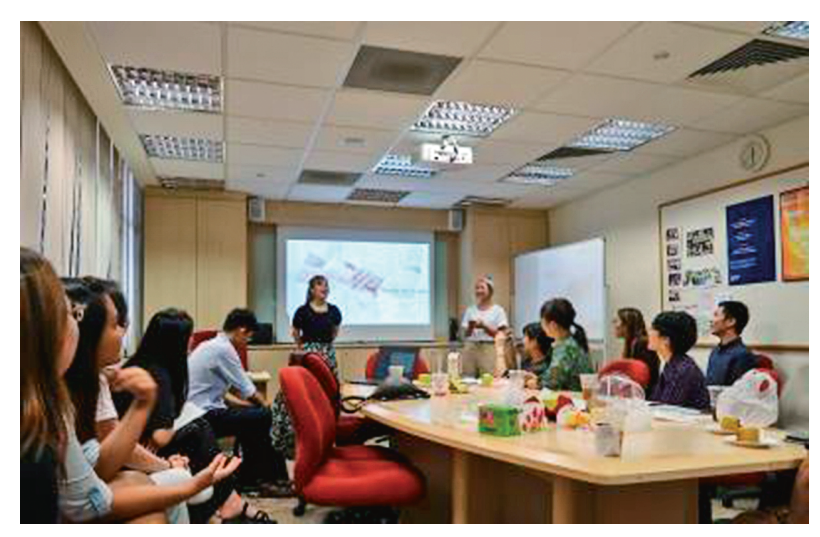

Figure-2 FILA student presentation at National University of Singapore

Course in Singapore. In September 2019, students from the FILA and Health Science and Nursing visited Singapore for a week (Figure-2). The field trip was led by Professor Yoko Okita. The program began in 2016 and has been conducted once a year since then. FILA students attended lectures about health in English and enhanced their mutual understanding with National University of Singapore students through international exchanges.

Juntendo University also runs the Japaneselanguage Immersion Program. The program is designed for overseas students who already speak some Japanese. Both FILA and Singaporean students have the opportunity to discuss various topics together while enjoying a rich cultural experience, such as Kabuki at the National Theatre of Japan. Students go on a field trip to one of the best summer resort areas in Japan. They gain indepth experience of other countries' cultures by visiting communities and facilities they could never see ordinarily.

\section{3) Customized learning for medical interpreters}

Starting in their 3rd year, FILA students select one area of interest on which to focus; it carries a total of 16 course credits. To provide an understanding about the issues and current conditions of global society, the FILA has established three different areas of research: Global Society; Inter-cultural Communication; and Global Health Services. For each area, there is a mandatory introductory course.

In Global Society, students learn about various global problems connected with a sustainable future, including economic globalization, international relations, energy conservation, food and water security, changes in weather patterns, and animal and plant diversity. In Global Health Services, students learn about the human body, health, bioethics, causes of diseases, international infectious diseases, safety of medicinal products, and health-care systems as well as various problems facing Japan and other countries, including rapidly aging societies. In Inter-cultural Communication, students learn about how to contribute to developing a multicultural, multilingual society; they learn how to do so through a rich understanding of the problems related to communication with foreign cultures by learning about people and culture, language and society, verbal and nonverbal communication, and translation and interpretation.

In their 3rd year, students can choose among a wide range of subjects according to their interests and career plans. In addition to patient care, they can study medical interpretation. The background of medical interpreting can be learned in various classes, such as English for Nursing Care taught by professors from Juntendo University Faculty of Health Care and nursing, English for Medical Care, Learning Sports in English, and Mechanism of Diseases taught by professor from Juntendo University Faculty of Medicine. Interfaculty collaboration is implemented in terms of education and some subjects contributes to develop skills needed for medical interpreters. For example, English for Medical Care provides basic and advanced knowledge and practical training to acquire five competencies for medical interpreters: (1) maintaining accuracy and completeness; (2) medical terminology and understanding the human body; (3) behaving ethically and making ethical decisions; (4) nonverbal communication skills; and (5) crosscultural communication skills using MHLW references ${ }^{9)}$.

To develop accuracy and completeness (1), students conduct role-play practice with scenarios that involve typical conversations in hospitals discussion, where the emphasis is placed on medical interpreting. With respect to medical terminology and understanding the human body (2), students play various games (such as an online matching game which matches English and Japanese medical 


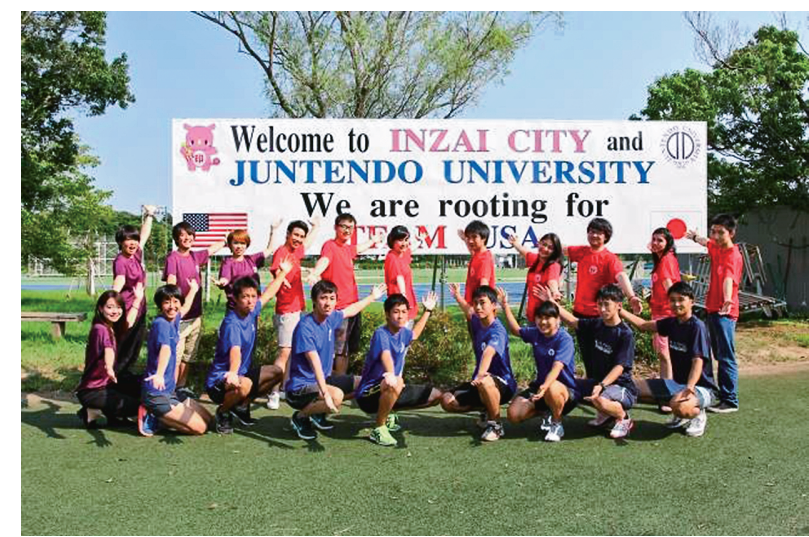

Figure-3 FILA student interpreters at the international athletics meeting in the city of Inzai

terms) related to medical terminology and basic anatomy. To address behaving ethically and making ethical decisions (3), students read handouts that deal with ethical considerations; discussions take place after lectures. Further, students reflect on their own perceptions and attitudes toward specific situations (such as how they should react if a patient gives them fruit in gratitude) by writing reports. To develop nonverbal communication skills (4), students take videos of one another while medical interpreting. They then review the videos to check their own nonverbal communication skills. The students also read about acquiring specific nonverbal communication skills and consider how to act as reliable medical interpreters in terms of nonverbal communication. For cross-cultural communication skills (5), the students read documentation about perceptions of symptoms and health care. For examples, the students read, in addition, the students make group presentations about patient culture with respect to specific ethnicities. The students enhanced their cross-cultural communication skills by reading and translating a guidebook to provide details on special points to consider when treating Muslim patients and by discussing cross-cultural communication.

\section{4) Practical opportunities}

Students from the FILA have acted as volunteer interpreters at international athletics meetings hosted jointly by the Faculty of Health and Sports Science of Juntendo University and the city of Inzai, in Chiba Prefecture (Figure-3). In that way,

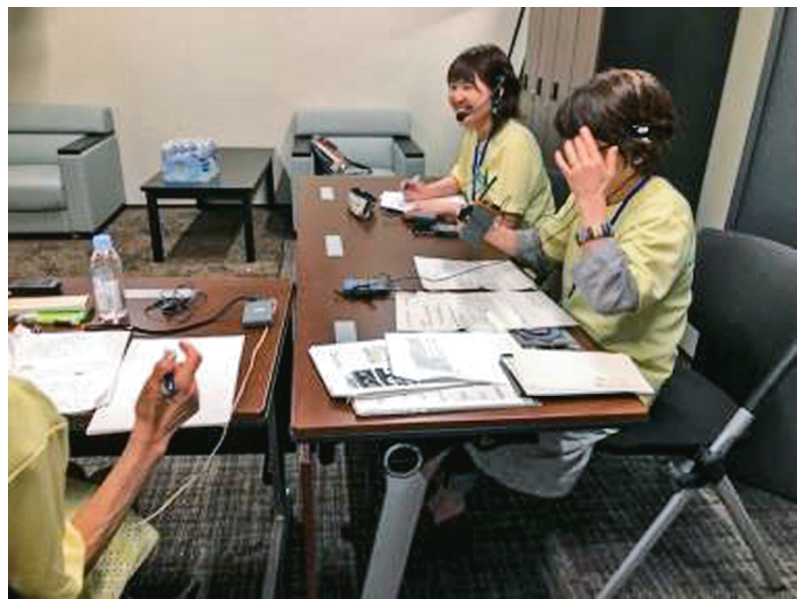

Figure -4 FILA students after interpreting at the Critical Link International 9 Conference

students could practice what they had learned through their daily studies in the actual settings of sporting events.

Students also have the chance to engage in practical training at Juntendo University Hospital in learning how to communicate with sick and handicapped patients. As shown in Figure-4, they had an opportunity to interpret at the closing ceremony of an international academic conference for medical interpreting held in Tokyo in June 2019: the 9th International Critical Link Conference, which was attended by representatives from every sphere of the interpreting community. The conference gives academics, interpreters, employers, trainers, policy makers, service providers, and service recipients the opportunity to participate in a lively, stimulating international exchange of information, knowledge, and skills. The Critical Link International Conference is held every 3 years and is a highly respected, global platform for interpreters, researchers, decision makers, academics, and clients of interpreters. The conference was first held in Canada in 1995. The 2019 conference attracted over 200 people and provided networking opportunities for participants from all over the world. The present author was a vice-chair at the Tokyo conference, and 17 FILA students worked as volunteers. The students engaged as simultaneous interpreters in the closing ceremony, introduced Japanese culture in a preconference tour, and also worked as bilingual receptionists. They had the opportunity to listen to keynote speeches, which offered an insight into state-of-the-art medical 
interpreting.

\section{Future perspectives: interfaculty collaboration}

Though only 5 years old, the FILA is backed by the traditions and infrastructure for international studies established at the first educational institution for Western medicine in Japan. Juntendo University has six departments: Medicine; Health and Sports Science; Health Care and Nursing; Health Science and Nursing; International Liberal Arts; and Health Science (Physical Therapy and Radiological Technology). Juntendo University is the only university in Japan that teaches students to become medical professionals in various fields as well as to become experts in the arena of sports. Interfaculty cooperation is needed to bridge the gap between medical professionals and patients with limited Japanese proficiency. By nurturing medical interpreters, Juntendo University is expected to promote interfaculty collaboration in supporting nonJapanese patients. As Ikeda ${ }^{21)}$ notes, Tokyo 2020 will take place during the hot summer: the medical support system-including medical interpreting to make Japan safe for both athletes and foreign visitors - is a matter of urgency. The Olympics and Paralympics are expected to be a key milestone for Japan to create an environment in which people from overseas can visit the country without worrying about medical care.

\section{Acknowledgments}

I thank the Edanz Group (www.edanzediting. com/ac) for editing a draft of this manuscript.

\section{References}

1) Japan National Tourism Organization: The trend of foreign guests from abroad to Japan. Monthly estimate. Retrieved from https://www.jnto.go.jp/jpn/statistics/vi sitor_trends/ (Last accessed at Feb. 7, 2020)

2) Statistics Bureau of Japan: The proportion of foreigners to Japanese in Japan exceeded $1 \%$ in 2000 . Retrieved from https://www.stat.go.jp/data/kokusei/2005/gaikoku/00/ 01.html (Last accessed at Feb. 7, 2020)

3) Schouten BC, Meeuwesen I: Cultural differences in medical communication: a review of the literature. Patient Educ Couns, 2006; 64: 21-34.

4) A fact sheet on the previous Olympic Games (2012). Fact sheet from previous Olympic Games (2014, September 20). Retrieved from http://www.olympic.org/london-20 12/documents-reports-studies-publications

5) Preuss H: Financing source development of mega sport events, paper presented at the 6th Annual Congress of the European College of Sport Science. Cologne 24-28, July 2001.

6) Ono N, Kurihara T: Interpreting at the Olympics: A survey of the literature and interview. Educational Studies, 2015; 57: 129-136.

7) Yue J: Sports interpreting in the Olympics. Proceedings of 2008 XVIII FIT (International Federation of Translators) World Congress. Shanghai, 2008.

8) Nishikawa C: Volunteering experience in Sochi Olympic Games (2014, September 20), 2014. http://www.ssf.or. jp/topics/sochi/tabid/712/Default. aspx (Last accessed at Feb. 7, 2020)

9) Ministry of Health, Labour and Welfare (2019): References regarding medical interpreting. Retrieved from https: //www.mhlw.go.jp/stf/seisakunitsuite/bunya/00000569 44.html (Last accessed at Feb. 7, 2020)

10) Ono N, Kiuchi T, Ishikawa H: Development and pilot testing of a novel education method for training medical interpreters. Patient Educ Couns, 2013; 93: 604-611.

11) Gany FM, Gonzalez CJ, Basu G, et al: Reducing clinical errors in cancer education: interpreter training. J Cancer Educ, 2010; 25: 560-564.

12) Pham K, Thornton JD, Engelberg RA, Jackson JC, Curtis JR: Alterations during medical interpretation of ICU family conferences that interfere with or enhance communication. Chest, 2008; 134: 109-116.

13) Lee SB: The socio-legal and training landscape of healthcare interpreting in Korea: From the viewpoint of medical tourism. J Trans Studies, 2009; 10: 115-154.

14) White K, Laws MB: Role exchange in medical interpretation. J Immigr Minor Health, 2009; 11: 482-493.

15) Espondaburu L: Interpreting bad news: What interpreters might learn from medical training and research. The ATA Chronicle, 2009; 13: 12-18.

16) Pugh MA, Vetere A: Lost in translation: an interpretative phenomenological analysis of mental health professionals' experiences of empathy in clinical work with an interpreter. Psychol Psychother, 2009; 82 (Pt 3): 305321.

17) Cross WM, Bloomer MJ: Extending boundaries: Clinical communication with culturally and linguistically diverse mental health clients and carers. Int J Ment Health Nurs, 2010; 19: 268-277.

18) Larrison CR, Velez-Ortiz D, Hernandez PM, Piedra LM, Goldberg A: Brokering language and culture: can ad hoc interpreters fill the language service gap at community health centers? Soc Work Public Health, 2010; 25: 387-407.

19) Norris WM, Wenrich MD, Nielsen EL, Treece PD, Jackson JC, Curtis JR: Communication about end-of-life care between language-discordant patients and clinicians: insights from medical interpreters. J Palliat Med, 2005; 8: 1016-1024.

20) Karliner LS, Perez-Stable EJ, Gildengorin G: The language divide: The importance of training in the use of interpreters for outpatient practice. J Gen Intern Med, 2004; 19: 175-183.

21) Ikeda H: The present state of medical support system in football and efforts directed at Tokyo 2020. Juntendo Medical Journal, 2018: 64: 437-443. 